\title{
A NEW SERIES OF CONJECTURES AND OPEN QUESTIONS IN OPTIMIZATION AND MATRIX ANALYSIS
}

\author{
JEAN-BAPTISTE HIRIART-URRUTY ${ }^{1}$
}

\begin{abstract}
We present below a new series of conjectures and open problems in the fields of (global) Optimization and Matrix analysis, in the same spirit as our recently published paper [J.-B. HiriartUrruty, Potpourri of conjectures and open questions in Nonlinear analysis and Optimization. SIAM Review 49 (2007) 255-273]. With each problem come a succinct presentation, a list of specific references, and a view on the state of the art of the subject.
\end{abstract}

Mathematics Subject Classification. 15A, 26B, 49K, 65C, 65K, 90C.

Received July 23, 2007. Revised February 4, 2008.

Published online June 24, 2008.

\section{INTRODUCTION}

"Some problems open doors, some problems close doors, and some remain curiosities, but all sharpen our wits and act as a challenge and a test of our ingenuity and techniques". So said Atiyah in the preface to Mathematics: frontiers and perspectives (2000, by the International Mathematical Union, published by the American Mathematical Society). This statement is, in our opinion, a good introduction to what a collection of problems is or should be. In mathematics, each area or subarea produces its own lists of (more or less celebrated) problems and open questions, sometimes hard to appreciate or just to understand if one does not work in the concerned field, as given evidence by the lists of problems offered in the above-referenced book.

Our objective here is more modest: we expose a selected list of questions that all belong to Optimization or Matrix analysis. They are of unequal importance and diverse origins, but all of rather wide interest. Some have a theoretical flavour, some others clearly hinge on calculation, and what it is asked for each of them varies. There are problems about which we know practically nothing, some others have partial answers; and also some are already solved but in rather indirect manners; for them we would like to have more natural, or at least different, proofs.

For each of the nine problems described in this paper, we propose a short presentation, the state of the art and a list of appropriate references. As a result, each of the problems listed can be read independently of the others, according to the interest or knowledge of the reader. The reader could thus try to tackle some of them, that is at least our aim in writing down such a paper.

\footnotetext{
Keywords and phrases. Convex sets, positive (semi)definite matrices, variational problems, energy functions, global optimization, permanent function, bistochastic matrices, normal matrices.

${ }^{1}$ Institut de Mathématiques de Toulouse, Université Paul Sabatier, 118 route de Narbonne, 31062 Toulouse Cedex 9, France. jbhu@cict.fr
} 
About notation: $\langle.,$.$\rangle denotes the usual inner product in \mathbb{R}^{n}$ and $\|$.$\| the associated norm; \operatorname{co}(S)$ stands for the convex hull of the set $S ; \operatorname{tr}(M)$ means the trace of the matrix $M$. The other used notations are standard ones in the fields of Optimization and Matrix analysis.

\section{List of problems.}

Problem 1. Progressive partial convexification of a set.

Problem 2. A generalized multilinear version of the Cauchy-Bouniakovski-Schwarz inequality.

Problem 3. Curves of minimal length in the plane or in the space.

Problem 4. Minimization of an energy for a general Coulombian problem.

Problem 5. Nonconvex global minimization of energy functions between particles.

Problem 6. Global minimization of the permanent function over the set of bistochastic matrices.

Problem \%. The Bessis-Moussa-Villani conjecture.

Problem 8. Conjecture on the lower bound of the inner product of sign vectors and unit vectors.

Problem 9. Conjecture on the determinant of normal matrices.

\section{Problem 1. Progressive partial Convexification of a Set}

Let $S$ be a subset of $\mathbb{R}^{n}$ and $p$ an integer $\geq 1$. Define the set $S_{p}$ as follows:

$$
S_{p}:=\left\{\sum_{i=1}^{p} \lambda_{i} x_{i}: x_{i} \in S, \lambda_{i} \geq 0 \text { for all } i, \sum_{i=1}^{p} \lambda_{i}=1\right\}
$$

Clearly $S_{1}=S, S_{1} \subseteq S_{2} \subseteq \ldots \subseteq S_{n} \subseteq S_{n+1}$, and this nested sequence of sets stops at the $(n+1)$-th step since $S_{n+1}$ is the convex hull coS of $S$ (Carathéodory's theorem).

The $S_{p}$ are "partially convexified forms" of $S$. A refinement of Carathéodory's theorem, due to Fenchel and Bunt (see [33], p. 99 and comments p. 403), states that $S_{n}=\operatorname{co} S$ if $S$ has at most $n$ connected components.

Question: What topological properties do the $S_{p}$ 's share?

Clearly, each $S_{p}$ is compact whenever $S$ is compact. Further, the $S_{p}$ 's are arcwise connected for $p \geq 2(n$ steps make $S_{2}$ pass to $S_{n+1}=\operatorname{coS} S_{2}$, which is another illustration of the Fenchel-Bunt theorem). The only result that we know, concerning the link between $S_{p}$ and coS ([26], Thm. in p. 590) is far from the question above.

A referee suggested that the variant of this problem for cones is also interesting since then the convex hull can be replaced by the sum: $K+K$, etc.

\section{Problem 2. A generalized multilinear Version of the Cauchy-Bouniakovski-Schwarz InEQuAlity}

Let $A$ and $M$ be two symmetric real $(n, n)$ matrices, with $A$ positive semidefinite; if

$$
|\langle M x, x\rangle| \leq\langle A x, x\rangle \text { for all } x \in \mathbb{R}^{n}
$$

then

$$
(\langle M x, y\rangle)^{2} \leq\langle A x, x\rangle \cdot\langle A y, y\rangle \text { for all } x, y \text { in } \mathbb{R}^{n} .
$$

This is a generalization of the classical Cauchy-Bouniakovski-Schwarz inequality. There is a generalized multilinear version, reading as follows: Let $k$ be an integer greater than 2 , let $H$ be a symmetric $k$-linear form on $\mathbb{R}^{n}$, let $A$ be a symmetric positive semidefinite $(n, n)$ matrix. Assume that

$$
|H(x, \ldots, x)| \leq(\langle A x, x\rangle)^{\frac{k}{2}} \text { for all } x \in \mathbb{R}^{n}
$$


then

$$
\left|H\left(x_{1}, \ldots, x_{k}\right)\right|^{2} \leq \prod_{i=1}^{k}\left\langle A x_{i}, x_{i}\right\rangle \text { for all } x_{1}, \ldots, x_{k} \text { in } \mathbb{R}^{n} .
$$

This result is proved by induction in ([50], Appendix 1). Following a first circulation of this problem by us, we have been told that there is another proof due to Lojasiewicz, reported in ([10], Prop. 1); we however must confess it is hard to make a connection with what we are considering here. Anyway, the proofs should be given more of an optimization flavor. Therefore our question is: Can the above inequality be proven by techniques from Optimization (we think of optimality conditions, possibly of higher order than second order ones)?

\section{Problem 3. Curves of minimal length in the plane or in the space}

In many variational problems the unknown is a curve (in the plane $\mathbb{R}^{2}$ or in the space $\mathbb{R}^{3}$ ). The constraints then concern the curve, and the criterion to be minimized is the length of the curve. The one we are going to present has two versions: a 2-dimensional one, which is more or less known in the folklore of variational analysis, and a 3-dimensional one for which very little is known - if anything.

\subsection{Searching for a curve of minimal length in the plane}

Consider a boat, lost in the sea, whose captain knows that it is located at 1 mile from the shore, assimilated with a line, (that is what the measuring instruments indicate), but the fog is so heavy that he is unable to assess the direction to take. The boat moves at a constant speed and the objective is to touch the seaside as soon as possible; so the question is: What is the path of minimal length that the boat should follow to be sure to touch or meet the seaside? In mathematical terms: Given a circle centered at $\mathrm{O}$ of radius 1 , what is the curve of minimal length, drawn from the origin $\mathrm{O}$, touching or meeting any tangent line to the circle? By "a curve" we mean here "a continuous rectifiable curve".

The solution, if any, is not unique: indeed, any optimal curve, twisted around the origin $\mathrm{O}$ by an arbitrary angle, has the same length, hence remains optimal. The first attempt, proving at least the feasibility of the problem, is to move as follows: the boat leaves the origin following a ray, at the end of the ray ( 1 mile), it turns around the circle of radius 1 (see Fig. 1a); in doing so, it will have covered a distance of $(2 \pi+1) \approx 7.2832$ miles. Of course, this proposal is rough. One can do better. How? I posed the problem several times to my students in mathematics or engineering sciences. One of their typical answers is described in Figure 1b: the boat moves beyond the end of the radius, returns to the circle along a line-segment tangent to the circle, follows half the circle, and the path ends with another line-segment tangent to the circle; the angle delimited by the radius followed when leaving the origin $\mathrm{O}$ and the one pointing towards the arrival point is twice $45^{\circ}$. Not so bad! In fact, a boat pursuing this path is sure to meet or touch any shore situated at 1 mile from the origin (starting point), wherever it be! The length of the trajectory is $(\pi+2+\sqrt{2}) \approx 6.5556$ miles.

But one can do better in the same style. Consider an angular sector $S_{\theta}$ with an angle between 0 and $45^{\circ}$ (see Fig. 1c), and let us determine the value of $\theta$ minimizing the length of the corresponding curve $C_{\theta}$. The length $L(\theta)$ of $C_{\theta}$ is $\left(2 \pi-4 \theta+2 \tan (\theta)+\frac{1}{\cos (\theta)}\right)$ miles. It is (globally) minimized for $\theta_{\text {opt }} \approx 36.37^{\circ}$ and the corresponding length is $L_{\text {opt }} \approx 6.4589$ miles. The first proposal chose $\theta=0$, while the students proposed an angular sector with $\theta=45^{\circ}$. But is the latest shortest curve optimal? This problem was one of the examples that served as guide and motivation in the book [49]; the author stopped his discussion there at the proposal above...

After some search in the literature, we found the full answer to our problem in the reference [39] (see also Problem A30 in [12] for further variants): the proposed curve is drawn in Figure 2a (beware, the vertical segment out of the circle is not on the path, it is drawn here to mark the bounds of the path on the right); its length is $\frac{7 \pi}{6}+\sqrt{3}+1 \approx 6.3972$. The proof of optimality is lengthy, a dozen of pages with analytical and geometrical arguments specific to the plane - and a bit damned annoying, we must confess. So, we ask for a proof using techniques and results from Calculus of variations or Optimal control. 


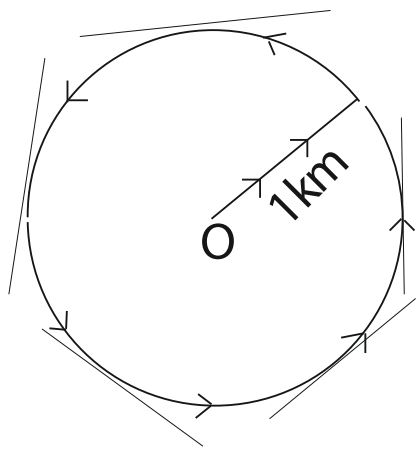

a

$$
\text { b }
$$

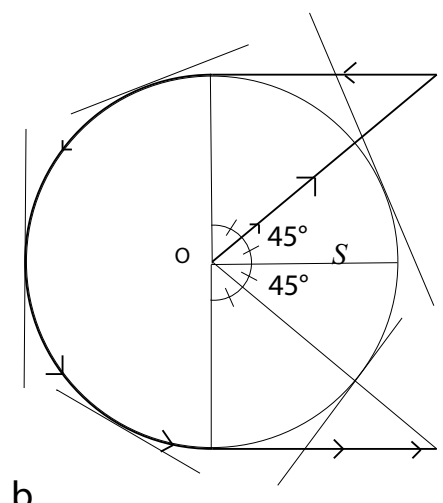

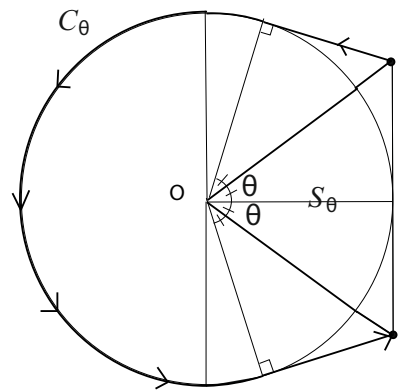

C

Figure 1. Searching for a curve of minimal length.
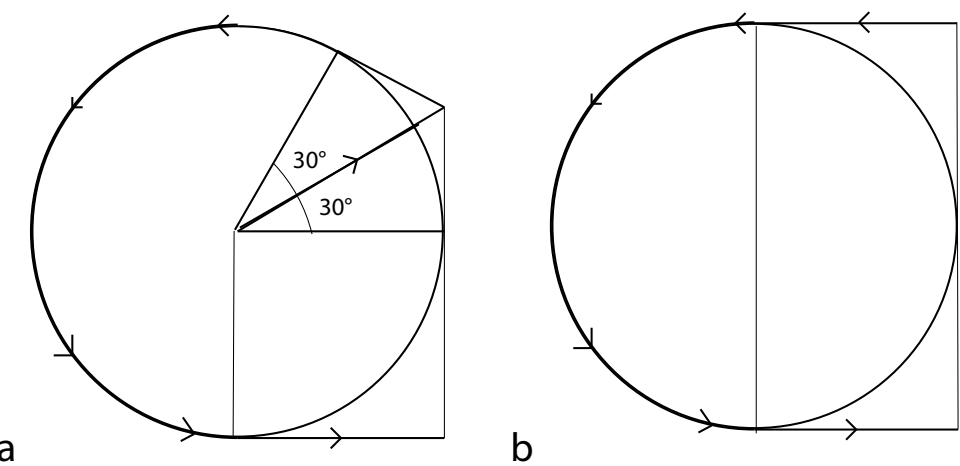

Figure 2. Optimal curves.

A related problem, a bit simpler, whose solution helps to understand the optimality of the curve in Figure 2a, is as follows. A telephone company has marked some spots on the ground to tell that its cable lies within 1 yard from the mark. A problem is pointed out to the telephone company: where the dispatched technician should dig, to be sure to find the cable, by digging the least possible (along a curve however)? This resembles to the preceding problem, except that there is no constraint on the departure point. The optimal trench turns out to be a half-circle extended with two line-segments like at the end of the preceding curve; see Figure $2 \mathrm{~b}$.

\subsection{Searching for a curve of minimal length in the space}

The problem tackled in Section 3.1 has a 3-dimensional version, no less interesting than the 2-dimensional one, but decidedly more difficult to handle: given a sphere centered at $\mathrm{O}$ of radius 1 , what curve, emanating from the origin $\mathrm{O}$, has minimal length and touches or meets each tangent plane to the sphere? Some of my colleagues have decorated this mathematical problem in terms of science fiction: an astronaut who left his space shuttle, knows he is at 10 yards distance from it, and he has to return back there; because the energy reserve at his disposal is bounded, he therefore has to pursue the shortest path in the space... Admittedly, we know nothing about the structure of the optimal path. Repeated considerations and collegian discussions have produced quite a few feasible solutions, some of rather strange shape. The present record (as of May 2006) is a curve whose length is 13.6699 times the length of the radius, that is to say $\approx 136.699$ yards in our example. 
We end with another problem, similar to the previous one, posed to us by Grigis (university of ParisVilletaneuse, France), while reacting to and wrestling with the above challenging problem: What is the curve of minimal length in the space which can be seen from any point on the earth? As one easily imagines, this problem has some practical applications: design of a laser neon tube for lighting any point of a sphere, find a trajectory for an artificial satellite so that any point on the earth could be observed, etc.

\section{Problem 4. Minimization of An energy for a general Coulombian problem}

As for most of the problems of this kind, the following originates in Physics [46,47]. It was posed to us by Ley and Mouchet (university of Tours, France): Find the geometrical configuration formed by $N$ particles so as to minimize a given energy function. More precisely, let $N$ distinct points (= particles) be given in the 3-dimensional space $\mathbb{R}^{3}, N \geq 3$. By choosing 3 points among these $N$ points, the considered set of points gives rise to $\left(\begin{array}{c}N \\ 3\end{array}\right)=\frac{N(N-1)(N-2)}{6}$ triangles, each having 3 angles. Whence the number of angles $\theta_{i}$ built up from the $N$ points is $\frac{N(N-1)(N-2)}{2}$, they will be the decision variables in our optimization problem. The so-called local energy function to be minimized is defined as $E_{N}=-\frac{1}{4}\left[\frac{N(N-1)}{2}+F_{N}\right]$, where

$$
F_{N}:=\sum_{\left\{\text {all angles } \theta_{i}\right\}} \cos \left(\theta_{i}\right) \text {. }
$$

The objective function $F_{N}$ depends only on the geometrical configuration of the $N$ points; it is invariant under the group of Euclidean isometries (rotations for example). Further, the scale independence of Coulombian interaction makes it also invariant under dilations as well. The optimization problem is now:

$$
\left(\mathcal{P}_{N}\right)\left\{\begin{array}{c}
\text { Maximize } F_{N} \text { over all the configurations formed from the } N \text { points, } \\
\text { subject to the } \frac{N(N-1)(N-2)}{6} \text { linear constraints } \theta_{i}+\theta_{j}+\theta_{k}=\pi .
\end{array}\right.
$$

The constraints in the above formulation express that $\theta_{i}, \theta_{j}, \theta_{k}$ are three angles in the same triangle. However, to get "true" triangles, one should check that the components $\bar{\theta}_{i}$ of an optimal $\bar{\theta}=\left(\bar{\theta}_{1}, \ldots, \bar{\theta}_{n}\right)$ in $\left(\mathcal{P}_{N}\right)$ are strictly positive.

We did not find this problem listed in the more or less classical geometrical optimization problems posed in $\mathbb{R}^{3}[1,51]$. The following partial results have been obtained in ([47], Sect. IV.B):

- When $N=3$. The global maximum of $F_{3}$, a function of 3 variables, is $\frac{3}{2}$; it is achieved when the three points form an equilateral triangle. This is an easy (still interesting) exercise in constrained optimization: first check that the optimization problem admits a solution, secondly prove that the components $\bar{\theta}_{1}, \bar{\theta}_{2}, \bar{\theta}_{3}$ of an optimal $\bar{\theta}$ are necessarily strictly positive, finally use the Lagrange multiplier rule to find the unique solution $\left(\frac{\pi}{3}, \frac{\pi}{3}, \frac{\pi}{3}\right)$.

- When $N=4$. The global maximum of $F_{4}$, a function of 12 variables, is 6 ; it is attained when the four points form a regular tetrahedron.

For larger $N$, numerical investigations led the author of [47] to the following three conjectures:

- When $N=5$. The global maximum of $F_{5}$, a function of 30 variables, is $\simeq 14.59$, and attained when the five points make two mirror-symmetric tetrahedrons sharing one common equilateral basis, the other faces being 6 isoceles identical triangles. In this problem, the pyramidal configurations with a squared basis leads to a local maximum of $F_{5}$, the corresponding value is $\simeq 14.57$.

- When $N=6$. The global maximum of $F_{6}$, a function of 60 variables, is $\simeq 28.97$; it is achieved when the six points make a regular octahedron.

- When $N=8$. The global maximum of $F_{8}$, a function of 168 variables is $\simeq 79.50$. It is achieved when the eight points form two identical squares (whose edges could be set of length one, without loss 
of generality) lying in two parallel planes separated by a distance $\simeq 0.97$; the axis joining the centers of the two squares is perpendicular to the squares and the two squares are twisted one from the other by an angle of $45^{\circ}$. In this problem, the cube-configuration yields the value 79.39 for the function $F_{8}$.

For a given collection of $N$ points, clustering the sum (4.1) in $M$-uplets (still $M \geq 3$ ) allows us to decompose $F_{N}$ into contributions of $M$-clusters as follows:

$$
F_{N}=\sum_{M_{i} \in\{M \text {-subclusters }\}} \frac{(N-M) !(M-3) !}{(N-3) !} F_{M_{i}} .
$$

Explanation: The same angle $\theta_{i}$ is common to $\left(\begin{array}{c}N-3 \\ M-3\end{array}\right)=\frac{(N-3) !}{(N-M) !(M-3) !}$ subclusters of $M$ points (once one has chosen an angle, that is 3 points, one has to choose $M-3$ points among $N-3$ points). By uniformly distributing the sum of cosines over all the possible $M$-clusters, one has to divide each cosine by the number of subclusters of $M$ points; whence the factor appearing in the formula (4.3). The sum in (4.3) involves $\left(\begin{array}{c}N \\ M\end{array}\right)$ terms, the number of $M$-clusters that can be built up from a collection on $N$ points.

As a consequence, it readily comes from (4.2):

$$
\sup F_{N} \leq \frac{N(N-1)(N-2)}{M(M-1)(M-2)} \sup F_{M}
$$

(the involved factor is $\left(\begin{array}{c}N \\ M\end{array}\right)$ times $\left.\frac{(N-M) !(M-3) !}{(N-3) !}\right)$; this induces the following string of inequalities:

$$
\frac{\sup F_{N}}{N(N-1)(N-2)} \leq \frac{\sup F_{N-1}}{(N-1)(N-2)(N-3)} \leq \frac{\sup F_{M}}{M(M-1)(M-2)} \leq \ldots \leq \frac{\sup F_{3}}{3.2 .1}=\frac{1}{4} .
$$

For example, when considering the identity (4.3) for $N=4$ and $N=3$, the optimal configurations for sets of 3 points give rise to optimal configurations for sets of 4 points (see the results above): the faces of the regular tetrahedron, that are equilateral triangles, maximize the contributions of all the 3 -subclusters simultaneously. In the string of inequalities above, equality actually holds for $N=3$ and $N=4$; the inequalities are tight for $N=5$, with a difference of less than $4 \%$, if the conjectured configuration is the right one.

Solving the optimization problem $\left(\mathcal{P}_{N}\right)$ with accuracy seems out of reach for larger $N$; this is a first challenge. As interesting as the approximate resolution of $\left(\mathcal{P}_{N}\right)$ for some values of $N$, is the asymptotic behavior of the optimal value and solution sets of $\left(\mathcal{P}_{N}\right)$ when $N \rightarrow+\infty$. In that respect, the following general conjecture was posed in [47]:

When $N \rightarrow+\infty$, the configurations that solve $\left(\mathcal{P}_{N}\right)$ correspond to

$$
N \text { points uniformly distributed on a sphere, and: } \sup F_{N} \sim \frac{2}{9} N^{3}+o\left(N^{3}\right) \text {. }
$$

This conjecture resonates with the celebrated Fekete optimization problem - to be reconsidered in the next section.

Incidentally, minimizing $F_{N}$ offers less interest; actually the global minimum of $F_{N}$ over all the possible configurations is obtained when all the $N$ points lie on the same line, the corresponding minimal value is $\frac{N(N-1)(N-2)}{6}$.

A problem similar to $\left(\mathcal{P}_{N}\right)$ is considered in [2]. The authors define there another geometrical multi-particle energy, whose expression is somehow different from $F_{N}$ (it is defined via an ad hoc "volume" function built up from the $N$ points), but coinciding with it when $N=3$; they ended up with the same questions as those posed here for our problem $\left(\mathcal{P}_{N}\right)$ (Sect. 5 in [2], more specifically). 


\section{Problem 5. Nonconvex global minimization of Energy functions BETWEEN PARTICLES}

Many famous - and among them some ancient - optimization problems originated in Physics, Chemistry or Mechanics. The ones considered here share some properties like the following ones: (i) they deal with nonconvex energy objective functions; (ii) the global minimizers are the ones of interest; (iii) more conjectures than proven results are known; (iv) they offer recurrent challenges for testing sophisticated global optimization routines. Next we present some of them, adding a name when they bear one.

Given a unit-sphere in $\mathbb{R}^{3}$, the generic problem is to figure out how a family of $N$ electrons (= points) would distribute themselves so as to minimize a total potential energy $V$ (to be defined). We denote by $\|$.$\| the usual$ Euclidean norm in $\mathbb{R}^{3}$, and $x_{1}, \ldots, x_{N}$ the $N$ distinct points in $\mathbb{R}^{3}$ (beware, $x_{i}$ is not the $i$ th coordinate of a point).

- The global optimization problem of M. Fekete (which dates back to 1923):

$$
\left(\mathcal{Q}_{N}\right)\left\{\begin{array}{l}
\text { Maximize } G_{N}\left(x_{1}, \ldots, x_{N}\right):=\prod_{1 \leq i<j \leq N}\left\|x_{i}-x_{j}\right\| \text { over all } \\
\text { the configurations formed from the } N \text { points on the sphere. }
\end{array}\right.
$$

Squaring the norm makes the objective function differentiable, but does not help in solving the problem. Since the $x_{i}$ are constrained to lie on the unit-sphere, $\left\|x_{i}-x_{j}\right\|^{2}=2\left(1-\cos \theta_{i, j}\right)$, where $\theta_{i, j}:=\arccos \left\langle x_{i}, x_{j}\right\rangle$; this is another way of expressing the objective function. Sometimes, $\left(\mathcal{Q}_{N}\right)$ is stated in the following equivalent form:

$$
\left(\mathcal{Q}_{N}\right)\left\{\begin{array}{l}
\text { Minimize } V_{N}\left(x_{1}, \ldots, x_{N}\right)=-\log G_{N}\left(x_{1}, \ldots, x_{N}\right)=\sum_{1 \leq i<j \leq N} \log \frac{1}{\left\|x_{i}-x_{j}\right\|} \\
\text { over all the configurations formed from the } N \text { points on the sphere. }
\end{array}\right.
$$

The solutions are called the (logarithmic or) elliptic Fekete points (of order $N$ ). The objective function $V_{N}$ represents (in Physics) the energy of $N$ charged particles that repel each other according to Coulomb's law; the objective is to have physically stable, minimal energy configurations.

For example, for $N=4$, the Fekete points form a tetrahedron. Exact solutions indeed are known for $N$ up to 6. But, already in case of $N=8$ points, intuition fails: the elliptic Fekete points do not form a cube. A cube where, for example, the upper plane is rotated over $45^{\circ}$ with respect to the bottom plane, gives already a larger value of $V_{N}$.

- The global optimization problem of P.M.L. Tammes (1930):

$$
\left(\mathcal{R}_{N}\right)\left\{\begin{array}{l}
\text { Maximize } H_{N}\left(x_{1}, \ldots, x_{N}\right):=\min _{1 \leq i<j \leq N}\left\|x_{i}-x_{j}\right\| \text { over all } \\
\text { the configurations formed from the } N \text { points on the sphere. }
\end{array}\right.
$$

Tammes was a Dutch botanist who encountered this problem when studying the pores in spherical pollen grains. $\left(\mathcal{R}_{N}\right)$ is closely linked to some hard "packing problems" in Combinatorial optimization like: how to pack $N$ identical circular caps on the surface of an unit sphere so that the diameter of the caps is as large as possible. An additional difficulty with this problem is that the objective function is not differentiable everywhere; smoothness is upset by the min operation appearing in its definition. Exact solutions are known for $N$ up to 12 and some very specific values like $N=24$.

- The global optimization problem of J.J. Thomson (1921):

$$
\left(\mathcal{S}_{N}\right)\left\{\begin{array}{l}
\text { Minimize } V_{N}\left(x_{1}, \ldots, x_{N}\right)=I_{N}\left(x_{1}, \ldots, x_{N}\right):=\sum_{1 \leq i<j \leq N} \frac{1}{\left\|x_{i}-x_{j}\right\|} \text { over } \\
\text { all the configurations formed from the } N \text { points on the sphere. }
\end{array}\right.
$$


A bit more general situation arises when the objective function to be minimized depends on $p>0$ as follows: $I_{N}^{p}\left(x_{1}, \ldots, x_{N}\right):=\sum_{1 \leq i<j \leq N} \frac{1}{\left\|x_{i}-x_{j}\right\|^{p}}$. In this case, as $p \rightarrow+\infty$, with the number $N$ of points fixed, the energy $I_{N}^{p}\left(x_{1}, \ldots, x_{N}\right)$ is more and more dominated by the term involving the smallest of the distances between points; thus, in some sense, it becomes asymptotically equivalent to $\left(\mathcal{R}_{N}\right)$.

- The global optimization problem of L. Fejes Toth (1956):

$$
\left(\mathcal{T}_{N}\right)\left\{\begin{array}{l}
\text { Maximize } J_{N}\left(x_{1}, \ldots, x_{N}\right):=\sum_{1 \leq i<j \leq N}\left\|x_{i}-x_{j}\right\|^{q} \text { over } \\
\text { all the configurations formed from the } N \text { points on the sphere. }
\end{array}\right.
$$

Here, $q>0$; actually, the problem is of interest only for $q<2$; for $q \geq 2$ and $N$ even, solving $\left(\mathcal{T}_{N}\right)$ becomes easy: an optimal configuration is obtained by placing half the points concentrated at the north pole and the other half at the south pole. Even for $q=1,\left(\mathcal{T}_{N}\right)$ is a long-standing open problem in Convex geometry.

It is time to state some common results, observations and questions on these problems:

- Since these problems are of interest in Physics, Chemistry (especially Crystallography), a huge literature exists spread apart from the usual journals publishing mathematical results; a sample of chosen references is proposed at the end. All problems instances display a high degree of symmetry, induced by equivalence of points arrangements on the sphere. Counting the number of critical points in Thomson's problem and solving Fekete's global optimization problem are considered by Smale ([55], Problems 6 and 7) as two of the main challenging mathematical questions for the XXI-th century.

- For small $N$, say up to 5 , the optimal configurations in all the problems are either the same or look very similar (the usual regular polyhedrons).

○ "Massive multiextremality": Problems $\left(\mathcal{Q}_{N}\right)-\left(\mathcal{T}_{N}\right)$ have many local minimizers and saddle points; it is estimated that the number of distinct local minimizers (ignoring rotations and reflexions) growths exponentially with $N$, at least for certain subsequences of integers. Many local solutions are not global ones, and some of them are fairly close (by their values) to the "true" (most typically unknown, but conjectured) global solutions.

- Reference [54] presents a concise review of the theoretical background for several of the considered problems. Theoretical results include: upper and lower bounds for the optimal values; estimates on the optimal distributions of points; for example, in the Fekete problem, the optimal solutions $\bar{x}_{i}$ do satisfy $\left\|\bar{x}_{i}-\bar{x}_{j}\right\| \geq \frac{3}{5 \sqrt{N}}$ for $i \neq j$ ([53], Thm. 3.4); asymptotic results, i.e. estimates on the behavior of optimal values as $N \rightarrow+\infty$, rely on hard mathematical calculations (see $[41,53,54]$ ).

- As said, optimal configurations are known only for a handful of integers $N$. Indeed, despite the relative simplicity of the objective function and constraint set, certificates of global optimality are not available; usual first-order and second-order optimality conditions are easy to derive but of little value. More theoretical work should be done on the subject, probably in the spirit of necessary and sufficient conditions for global optimality such as obtained when one has to minimize a quadratic function over a constraint set defined (as an equality or inequality) by a single quadratic function.

- Test problems for "massive high precision computer experimentation". The evoked problems are wellsuited to testing global optimization routines (examples: [7,36,52,56]). Actually, numerical determination of solutions leads to rapidly growing computational demands, which can easily become prohibitive. Carrying out these procedures lead to stable solutions for, say, $N=$ some tens (depending on the problem tackled). The instance $N=150$ is already difficult, although attempts have been made for $N=50000$. In short, the precise determination of optimal configurations for large $N$ is yet out of reach; however explicit constructions of configurations that are proven to be close to optimal ones have been constructed.

- There is no reason to restrict the constraint set to being the sphere, some applied problems (molecular modeling of protein structures for example) require the constraint set being an arbitrary compact manifold $S$ in $\mathbb{R}^{3}$. Some other constraint sets like the torus, or non-smooth ones, like the unit-cube, are considered from the numerical viewpoint in the recent works $[3,7,28]$. 
- Unconstrained variants of the problems above are also of interest for physicists or chemists, as for example:

$$
\left(\widehat{\mathcal{S}_{N}}\right)\left\{\begin{array}{l}
\text { Minimize } \widehat{I_{N}}\left(x_{1}, \ldots, x_{N}\right):=\sum_{1 \leq i<j \leq N} \frac{1}{\left\|x_{i}-x_{j}\right\|}+\sum_{i=1}^{N}\left\|x_{i}\right\|^{2} \text { over } \\
\text { all the configurations formed from the } N \text { points in } \mathbb{R}^{3} .
\end{array}\right.
$$

In a certain sense, this a penalized or regularized version of problem $\left(\mathcal{S}_{N}\right)$; it also has a physical interpretation (the objective function to minimize is an electrostatic repulsion energy plus a mechanical attraction to the origin).

Another popular unconstrained global optimization problem, coming from the aim of designing the best protein folding structures is as follows:

- The Lennard-Jones problem:

$$
\left(\widehat{\mathcal{U}_{N}}\right)\left\{\begin{array}{l}
\text { Minimize } \widehat{K_{N}}\left(x_{1}, \ldots, x_{N}\right):=\sum_{1 \leq i<j \leq N}\left[\frac{1}{\left\|x_{i}-x_{j}\right\|^{12}}-\frac{2}{\left\|x_{i}-x_{j}\right\|^{6}}\right] \\
\text { over all the configurations formed from the } N \text { points in } \mathbb{R}^{3} .
\end{array}\right.
$$

The Lennard-Jones interaction energy seems to be a good model for rare gas microclusters (aggregate of small number of atoms). In a journal like Journal of Global Optimization, almost each issue contains a contribution to the solution of this kind of problem.

\section{Problem 6. Global minimization of the permanent function over the set OF BISTOCHASTIC MATRICES}

\subsection{From the conjecture of Van der Waerden to the theorem of Falikman and Egorychev}

Van der Waerden stated the following problem in 1926. For a matrix $A=\left[a_{i, j}\right]$, let per $A$ denotes the so-called permanent of $A$, that is

$$
\operatorname{per} A:=\sum_{\substack{\{\text { permutations } \sigma \\ o f}} \prod_{i=1}^{n} a_{i, \sigma(i)}
$$

(this is the little cousin of the determinant of $A$ ). Let $\mathcal{B}_{n}$ denote the set of positive bistochastic matrices (i.e., matrices with nonnegative entries and whose columns and rows sum up to 1 ; also called doubly stochastic matrices); particular matrices in $\mathcal{B}_{n}$ are permutation matrices (with only one 1 in each column and in each row, other entries being 0 ) whose permanents equal 1, and the "central" $J_{n}=\left[\frac{1}{n}\right.$ everywhere $]$ whose permanent is $\frac{n !}{n^{n}}$. Van der Waerden conjectured that

$$
\operatorname{per} A \geq \operatorname{per} J_{n} \text { for all } A \text { in } \mathcal{B}_{n} \text {, with equality if and only if } A=J_{n} \text {. }
$$

The proposed conjecture inspired a flood of papers by many authors who proved the statement for $n=$ $3,4,5$ and for many special subclasses of bistochastic matrices. Finally, the Van der Waerden conjecture was independently settled by two soviet mathematicians Falikman and Egorychev in $1981[17,18,20]$. Although the two proofs differ in details, they both use the same new idea: the main point is the reference to Alexandrov inequalities for the so-called mixed discriminants of quadratic forms (1937-1938). Actually, per $A$ is the mixed discriminant of a certain set of diagonal quadratic forms, and Alexandrov proved his inequalities in the process of giving a proof of better-known Alexandrov-Fenchel inequalities for mixed volumes of convex bodies. 
It is puzzling to observe that Van der Waerden's inequality is a constrained global optimization problem, and that the proposed proofs rely on cunning inequalities on quadratic forms... We therefore ask for a proof which would entirely belong to the optimization realm. Since the objective function per $A$ is a multilinear form as a function of columns and rows, we think that, like for Problem 2, optimality conditions, possibly of higher order than second order ones, must be invoked. The main difficulty here is that (6.1) is a constrained global optimization problem, even if the constraint set looks nice (a convex polyhedral set).

In order to help an enthusiastic reader who wants to try his luck, and save him getting lost in the jungle of papers devoted on the subject, we delineate below the main properties of the constraint set $\mathcal{B}_{n}$ and of the objective function per $A$.

- $\mathcal{B}_{n}$ is a compact convex polyhedral set of the vector space $\mathcal{M}_{n}(\mathbb{R})$. It is "flat" in the sense that its affine hull is of dimension $(n-1)^{2}$. Its relative interior $\left(i . e\right.$., interior in the affine manifold generated by $\mathcal{B}_{n}$ ) consists of positive bistochastic matrices whose entries all are positive. The orthogonal subspace to the affine hull of $\mathcal{B}_{n}$ as well as algorithms to find the matrix in $\mathcal{B}_{n}$ closest to a given matrix $A$ are depicted in [57]. Indeed, the function $\frac{1}{\prod_{1 \leq i, j \leq n} a_{i, j}}$ of $A=\left[a_{i, j}\right]$ was used by D.I. Falikman as an interior penalty function (or barrier function); his approach was heavily based on the minimization of the perturbed function

$$
\operatorname{per}_{\epsilon}(A):=\operatorname{per}(A)+\epsilon \frac{1}{\prod_{1 \leq i<j \leq n} a_{i, j}}
$$

on the relative interior of $\mathcal{B}_{n}$; the additional term blows ups as $A$ approaches the relative boundary of $\mathcal{B}_{n}$.

The vertices of $\mathcal{B}_{n}$ are exactly the permutation matrices (there are $n$ !, making up the finite set $\Pi_{n}$ ), so that $\mathcal{B}_{n}$ is the convex hull of $\Pi_{n}$ (this is the celebrated Garrett Birkhoff's theorem, 1946). The relative boundary of $\mathcal{B}_{n}$, hence the various faces of $\mathcal{B}_{n}$, are much more complicated to describe, and this one of the main difficulties in optimizing on $\mathcal{B}_{n}$.

- About the permanent function:

- $\operatorname{per} A$ is a symmetric multilinear function (of columns and rows), positively homogeneous of degree $n$ (that is, $\operatorname{per}(t A)=t^{n} \operatorname{per} A$ for all $\left.t \in \mathbb{R}\right)$, but, contrary to its cousin $\operatorname{det} A$, there are numerical difficulties in evaluating it, and it fails to inherit two key properties of the determinants: the multiplicative property and the invariance under certain elementary operations on matrices. Moreover, for $n \geq 3$, it is impossible to transform a permanent into a determinant of a modified matrix: indeed, it is not possible to find a sequence $\left(\epsilon_{i j}\right)$ of elements in $\{-1,+1\}$ such that $\operatorname{per}\left(A_{\epsilon}\right)=\operatorname{det} A$ for all $A \in \mathcal{M}_{n}(\mathbb{R})$, where $A_{\epsilon}=\left[\epsilon_{i j} a_{i j}\right]$.

- As a continuous multilinear function, $\operatorname{per} A$ is a $C^{\infty}$-function of $A$, whose Taylor development (is exact) and stops at the $n$-th term; in other words the $(n+1)$-th differential function of the permanent function is zero everywhere (see (6.4) below). Concerning the other differentials, we have:

- for all $k \in\{1, \ldots, n\}$, the $k$-th differential $D^{k}$ per of the permanent function is homogeneous of degree $n-k$; - for $p \in\{1, \ldots, n-1\}$,

$$
\begin{gathered}
\frac{1}{(n-p) !} D^{n-p} \operatorname{per}(B) \underbrace{(A, \ldots, A)}_{n-p \text { times }}=\frac{1}{p !} D^{p} \operatorname{per}(A) \underbrace{(B, \ldots, B)}_{p \text { times }} \\
\frac{1}{n !} D^{n} \operatorname{per}(A) \underbrace{(B, \ldots, B)}_{n \text { times }}=\operatorname{per} A .
\end{gathered}
$$

$\circ \operatorname{per} A$ is not a convex function of $A$ (although it is not too far from being convex); if so was the case, (6.1) would have been a (nice) convex minimization problem. 
o Let $A(i, j)$ denotes the $(n-1, n-1)$ matrix obtained from $A$ by deleting the $i$-th row and the $j$-th column. Like for the determinant function, we have:

$$
\begin{gathered}
\operatorname{per} A=\sum_{i=1}^{n} a_{i, j} \operatorname{per} A(i, j)=\sum_{j=1}^{n} a_{i, j} \operatorname{per} A(i, j) \\
(\text { development following a column or a row); } \\
\operatorname{per}(A+t B)=\operatorname{per} A+t \sum_{i, j} b_{i, j} \operatorname{per} A(i, j)+O\left(t^{2}\right) . \\
\text { (first-order development) }
\end{gathered}
$$

From (6.3) we derive that the gradient of the permanent function at $A$ is the matrix whose $(i, j)$-th entry is $\operatorname{per} A(j, i)$ (working on the space $\mathcal{M}_{n}(R)$ made Euclidean with the usual inner product $\ll U, V \gg:=$ trace of $\left.U^{T} V\right)$.

○ Higher order Taylor developments:

$$
\operatorname{per}(A+t B)=\operatorname{per} A+\sum_{k=1}^{n} \frac{t^{k}}{k !} D^{k} \operatorname{per}(A) \underbrace{(B, \ldots, B)}_{k \text { times }} .
$$

$\circ \operatorname{det} A \leq \operatorname{per} A$ whenever $A$ is symmetric positive semidefinite. The conjectured inequality (6.1) was proved quite early for symmetric positive semidefinite matrices $A$ ( $c f$. [45]); by a happy combination of circumstances, that was the object of a long problem in a national competition for hiring teachers in France in 1980, just before the appearance of proof of the conjecture in the general case [5].

○ If $A \in \mathcal{B}_{n}, 0<\operatorname{per} A \leq 1$, and $\operatorname{per} A=1$ if and only if $A$ is a permutation matrix. The constrained optimization problem consisting in minimizing the per function on $\mathcal{B}_{n}$ does have solutions: these solutions cannot be permutation matrices, they lie on the remaining part of the relative boundary of $\mathcal{B}_{n}$ or on its relative interior. However, rather early, it became known that $J_{n}$ was the unique permanent-minimizing matrix not on the relative boundary of $\mathcal{B}_{n}$ ([44] and [45], Chap. 5).

○ The particular matrix $J_{n}$, located at the "center" of $\mathcal{B}_{n}$, enjoys some bizarre invariance property: $\operatorname{per}\left(J_{n}\right)=$ $\operatorname{per}\left(J_{n}(i, j)\right)$ (beware, $J_{n}(i, j)$ is not $\left.J_{n-1}\right)$. It happens that this invariance property $\operatorname{per}(A)=\operatorname{per}(A(i, j))$ characterizes the minimizers $A$ of per on $\mathcal{B}_{n}$, and that was the key ingredient which led to the proofs of the Van der Waerden conjecture $[40,42,45,58]$.

There still are several conjectures and open problems concerning permanents of matrices (see the survey [11]); among them we retain one which is very close, in its formulation, to the one exposed above in (6.1): The permanent function on the set $B_{n} \cap\{A: \operatorname{tr}(A)=0\}$ achieves its minimum uniquely at the matrix all of whose off-diagonal are $\frac{1}{n-1}$.

\subsection{Extensions}

It turns out that there are analogs or extensions of Van der Waerden optimization problem, sometimes connecting unexpectedly different areas of mathematics. One of them is Bapat's conjecture (1989), quite recently proved by Gurvits [23]. Let us expose it shortly.

Consider $n$ matrices in $\mathcal{M}_{n}(\mathbb{R})$. Then $\operatorname{det}\left(\sum_{i=1}^{n} t_{i} A_{i}\right)$ is a homogeneous polynomial of degree $n$ in the real variables $t_{i}$. The number

$$
D\left(A_{1}, \ldots, A_{n}\right):=\frac{\partial^{n} \operatorname{det}}{\partial t_{1} \ldots \partial t_{n}}\left(\sum_{i=1}^{n} t_{i} A_{i}\right)
$$


is called the mixed discriminant of $A_{1}, \ldots, A_{n}$. Among the various expressions of the $D$ function, we retain the following one

$$
D\left(A_{1}, \ldots, A_{n}\right)=\sum_{\substack{\{\text { permutations } \sigma \\ \text { of }\{1,2, \ldots, n\}\}}} \operatorname{det}\left(A_{\sigma}\right),
$$

where the matrix $A_{\sigma}$ is built up from $A_{1}, \ldots, A_{n}$ as follows: the $i$-th column vector of $A_{\sigma}$ is the $i$-th column vector of $A_{\sigma(i)}$. For an example, if $A_{1}=\ldots=A_{n}=A$, then $D\left(A_{1}, \ldots, A_{n}\right)$ is just $n ! \operatorname{det}(A)$.

Now, let $\mathfrak{B}_{n}$ denote the so-called set of positive semidefinite bistochastic $n$-uples of matrices $A_{1}, \ldots, A_{n}$, i.e. satisfying for each $i$ :

$$
\begin{gathered}
A_{i} \text { is positive semidefinite } \\
\text { trace of } A_{i}=1 \\
A_{1}+\ldots+A_{n}=I_{n} \text { (the identity matrix) }
\end{gathered}
$$

Bapat's conjecture [4] was:

The minimum value of $D\left(A_{1}, \ldots, A_{n}\right)$ over the set $\mathfrak{B}_{n}$ is $\frac{n !}{n^{n}}$;

it is attained uniquely for the positive semide finite $n$-uple $\frac{1}{n} I_{n}, \ldots, \frac{1}{n} I_{n}$.

The resemblance of (6.5) with (6.1) is striking - and more precise when one observes the following two points: firstly, if $A$ is a doubly stochastic matrix, the $n$-uple of matrices $A_{1}, \ldots, A_{n}$, where, for all $i, A_{i}$ is taken as the diagonal matrix with the $i$-th column (or row) vector of $A$ on the diagonal, clearly belongs to $\mathfrak{B}_{n}$; secondly, $D\left(A_{1}, \ldots, A_{n}\right)=\operatorname{per} B$ if again, for all $i, A_{i}$ is taken as the diagonal matrix with the $i$-th column (or row) vector of $B$ on the diagonal. However, the vertices of $\mathfrak{B}_{n}$ are not explicitly known except in some particular cases [4]. So, with what has been noticed earlier concerning $D(A, \ldots, A$,$) , the mixed discriminant can be viewed as a$ generalization of both the determinant and the permanent. As we said before, Bapat's conjecture was solved in [23] (even for complex Hermitian positive semidefinite matrices).

It seems that $\frac{n !}{n^{n}}$ is an universal minimum value for some complicated optimization problems. A further example is the next one. Consider a homogeneous polynomial function $p\left(z_{1}, \ldots, z_{n}\right)$ of degree $n$ in $n$ complex variables. Assume that this polynomial function satisfies the following property:

$$
\left|p\left(z_{1}, \ldots, z_{n}\right)\right| \geq \prod_{i=1}^{n} \operatorname{Re}\left(z_{i}\right)
$$

on the region $\left\{\left(z_{1}, \ldots, z_{n}\right) \in C^{n}: \operatorname{Re}\left(z_{i}\right) \geq 0\right.$ for all $\left.i=1, \ldots, n\right\}$.

Then, Gurvits [24] proves that

$$
\left|\frac{\partial^{n} p}{\partial z_{1} \ldots \partial z_{n}}\right| \geq \frac{n !}{n^{n}}
$$

In the proof, interesting connections are made with another topic, the one dealing with the so-called hyperbolic polynomials.

But, we wander from our initial question, of a more modest scope, that we repeat here: a proof of the inequality (6.1), entirely based on optimality conditions of higher order for constrained optimization problems.

\section{Problem 7. The Bessis-Moussa-Villani conjecture}

\subsection{The various forms of the Bessis-Moussa-Villani conjecture}

The next conjecture originated in a paper by the physicists Bessis, Moussa and Villani in 1975 [8], who attempted to simplify the calculation of the so-called partition functions of quantum mechanical systems. It refers 
to a positivity property of traces of matrices which, if verified, would allow the calculation of explicit error bounds in a sequence of approximations known as the Padé approximants. The conjecture seems important in areas of physics like condensed matter physics. Here is the statement of the BMV conjecture (as it is called in the literature):

Let $A$ and $B$ be Hermitian $(n, n)$ matrices with $B$ positive semidefinite. Then $\lambda \in \mathbb{R} \longmapsto \operatorname{tr}\left[\mathrm{e}^{(A-\lambda B)}\right]$ is the Laplace transform of a positive measure $\mu$ on $[0,+\infty)$ (i.e., it equals $\int_{0}^{+\infty} \mathrm{e}^{-\lambda x} \mathrm{~d} \mu(x), \mu$ depending on $A$ and $B$, of course).

The conjecture is known to be true when $A$ and $B$ commute (the proof is easy), as a general rule for $n=2$ (with an explicit construction of the corresponding measure $\mu$ ), but even for $n=3$, the complete answer is not known. By a classical theorem of Bernstein (sometimes called "the big Bernstein theorem"), (7.1) is equivalent to (7.2) below, asserting the "complete monotonicity" of some function:

$$
\text { Let } f(\lambda):=\operatorname{tr}\left[\mathrm{e}^{(A-\lambda B)}\right] ; \text { then for all positive integer } r,(-1)^{r} f^{(r)}(\lambda) \text { is nonnegative on }[0,+\infty) \text {. }
$$

Recent results on this question appeared in $[15,21,25,27,30,31,37,48] \ldots$ The most recent one [30] summarizes the state of the art for this conjecture. The current belief is that the BMV conjecture is true.

A rather recent equivalent formulation of (7.1) has been provided by Lieb and Seiringer [43]; it is more "palpable" than (7.1), we would say, since it is expressed as only positivity statements about coefficients of a family of polynomial functions. Here is this statement:

$$
\begin{aligned}
& \text { For all Hermitian positive semide finite }(n, n) \text { matrices } A \text { and } B, \\
& \text { and all positive integer } p \text {, the polynomial function } \\
& \qquad \begin{array}{c}
\lambda \in \mathbb{R} \longmapsto g(\lambda):=\operatorname{tr}\left[(A+\lambda B)^{p}\right]=\sum_{k=0}^{p} a_{k} \lambda^{k} \\
\text { has only nonnegative coefficients } a_{k} .
\end{array}
\end{aligned}
$$

Although not obvious, the polynomial function $g$ above has all real coefficients (indeed they are traces of Hermitian matrices); some of the factors whose sum give the coefficient of $\lambda^{k}$ may be negative, but what the conjecture says is that the sum of all these terms (summing up to the coefficient of $\lambda^{k}$ ) are nonnegative. The coefficients of $\lambda^{k}$ in $g$ have been proved to be nonnegative up to $k=5$ (and up to $k=6$ for the specific case where $n=3$ ) in [31]; after having written this paper we have been informed that the case $k=7$ is now achieved (in $[25]$ ).

Papers are published in a regular way on the BMV conjecture, in the physics literature as well as in the mathematics literature. Among the most recent (general) results on the subject, we retain the following one from [30]: if the coefficient of $\lambda^{k_{0}}$ of the polynomial function $g$ is nonnegative, then all the coefficients of $\lambda^{k}$, for $k \leqslant k_{0}$, are nonnegative; it is therefore equivalent to have (7.3) for infinitely many positive integers $k_{0}$.

There are also some contributions from the "stochastic side": in [21], the authors showed that the conjecture holds in the average for some random choices of matrices; in [15] the case $n=3$ is solved but for specific matrices $A$ and $B$.

As written in [48], "even if the applications are apparently limited, the BMV conjecture remains mathematically interesting, due to its simplicity, and due to the numerous unsuccessful attempts which have been performed to its resolution".

\subsection{A generalization: The "positivity conjecture"}

For a $(n, n)$ matrix $M$, let $S_{m, p}(M)$ denotes the sum of the $(m, m)$ principal minors of $M^{p}$; thus, given two matrices $A$ and $B, S_{m, p}(A+\lambda B)$ is a polynomial function of $\lambda$, of degree $m p$. 
The so-called "positivity conjecture" is as follows:

For all Hermitian positive semidefinite $(n, n)$ matrices $A$ and $B$, and all positive integer $p$, the polynomial function

$\lambda \in \mathbb{R} \longmapsto g_{m}(\lambda):=S_{m, p}(A+\lambda B)$

has only nonnegative coef ficients.

The coefficient of $\lambda^{k}$ in $g_{m}(\lambda)$ involves the sum of several matrices, a sum which turns out to be a Hermitian matrix, but not necessarily positive semidefinite (even if $A$ and $B$ are).

Let us look at the two extreme cases, for $m=1$ and for $m=n$. For $m=1,(7.4)$ is just the BMV conjecture. For $m=n$, this is an easy matter, a relation on determinants: all the coefficients of the polynomial function $\lambda \in R \longmapsto g_{n}(\lambda):=\operatorname{det}\left[(A+\lambda B)^{p}\right]$ are nonnegative ([38], Thm. 1).

The "positivity conjecture" (7.4) was solved for $n=2$ in [38].

\section{Problem 8. Conjecture on the lower Bound of the inner Product OF SIGN VECTORS AND UNIT VECTORS}

We discovered this conjecture in the field of Automatic control theory (when dealing with robust solutions of uncertain quadratic problems [6]), but it also appeared in other areas like Combinatorics and Probability theory [34]. Consider

- a unit vector $a=\left(a_{1}, a_{2}, \ldots, a_{n}\right)$ in $\mathbb{R}^{n}$ (i.e., $\|a\|^{2}:=\sum_{i=1}^{n} a_{i}^{2}=1$ );

- $n$ independent random variables $X_{1}, X_{2}, \ldots, X_{n}$, with the same distribution

$$
P\left(X_{i}=1\right)=P\left(X_{i}=-1\right)=\frac{1}{2}
$$

then

$$
P\left(\left|\sum_{i=1}^{n} X_{i} a_{i}\right| \leqslant 1\right) \geqslant \frac{1}{2}
$$

The range for the inner product $\sum_{i=1}^{n} X_{i} a_{i}$ is the line segment $[-\sqrt{n},+\sqrt{n}]$; what (8.1) asserts is that the value of this inner product is "concentrated" more than half the time on the small segment $[-1,+1]$. The lower bound $\frac{1}{2}$ is sharp, even for small dimensions (example, for $n=2$ ).

If one does not want to speak of probabilities, here is an equivalent way of formulating this conjecture. For $i=1, \ldots, n$ let $\varepsilon_{i} \in\{-1,+1\}$, so that the $2^{n}$ real numbers $\varepsilon_{1} a_{1}+\varepsilon_{2} a_{2}+\ldots+\varepsilon_{n} a_{n}$ are distributed over the line segment $[-\sqrt{n},+\sqrt{n}]$; then at least half of them lie in $[-1,+1]$ :

$$
\frac{\text { Card }\left\{\left(\varepsilon_{1}, \ldots, \varepsilon_{n}\right): \varepsilon_{i} \in\{-1,+1\} \text { for all } i, \text { and }\left|\sum_{i=1}^{n} \varepsilon_{i} a_{i}\right| \leqslant 1\right\}}{2^{n}} \geqslant \frac{1}{2} .
$$

Among the various interpretations of (8.2), in addition to Probability theory and Combinatorics, we retain the two following ones (from [34]):

- In terms of partitions. If we partition a sum $\sum_{i=1}^{n} a_{i}$ (with $\|a\|=1$ ) into two partial sums, then at least half of all partitions are roughly equal, i.e. they differ by at most 1: if

$$
\sum_{i=1}^{n} \varepsilon_{i} a_{i}=\sum_{i \in I^{+}} a_{i}-\sum_{i \in I^{-}} a_{i}, \quad I^{+} \subset\{1, \ldots, n\}, I^{-} \text {is the complementary set of } I^{+} ;
$$

then, according to (8.2),

$$
\sum_{i \in I^{-}} a_{i}-1 \leqslant \sum_{i \in I^{+}} a_{i} \leqslant \sum_{i \in I^{-}} a_{i}+1
$$

for at least half of the possibilities on $I^{+}$. 
- A geometrical interpretation. Consider an $n$-dimensional Euclidean ball and a smallest $n$-dimensional cube containing it. Then the conjecture asserts that for any pair of parallel supporting hyperplanes of the ball, at least half of the vertices of the cube lie between (or on) the two hyperplanes.

In [6], the authors proved the lower bound $\frac{1}{3}$ in (8.1) or (8.2). Actually a better lower bound was provided in [34]: $\frac{3}{8}$ (hence an improvement by about 4\%); the main tool in the proof is the following result which is interesting in its own right: if $X$ and $Y$ are independent real-valued random variables, each having a symmetric distribution with variance $\frac{1}{2}$, then $P(|X+Y|<1) \geq \frac{3}{8} \ldots$ So, still $12.5 \%$ is missing in the best known lower bounds to get at the conjectured $\frac{1}{2}$.

Reacting to a talk on these conjectures we delivered at the beginning of the year 2007, Haraux (University of Paris VI, personal communication on March 2007) was able to prove the following relaxed version of (8.1), namely:

$$
P\left(\left|\sum_{i=1}^{n} X_{i} a_{i}\right| \leqslant \sqrt{2}\right) \geqslant \frac{1}{2}
$$

Also a variant of (8.1) is proved in [13] (Lem. 27): the inequality (8.1) holds true if the random vector $\left(X_{1}, X_{2}, \ldots, X_{n}\right)$ is uniformly distributed over the sphere of $\mathbb{R}^{n}$ of radius $\sqrt{n}$.

There are further lower bounds which could derive from (8.1), for example: if $A$ is a $(n, n)$ symmetric matrix and the random vector $X=\left(X_{1}, X_{2}, \ldots, X_{n}\right)$ as for (8.1), then the following inequality was conjectured in [6]:

$$
P(\langle A X, X\rangle \leqslant \operatorname{tr} A) \geqslant \frac{1}{4} .
$$

The authors in [6] got at $\frac{1}{n^{2}}$ as a lower bound. The most recent results in that direction we are aware of come from putting together the results in [14] and [29]: the probability in (8.3) is bounded from below by $\max \left(\frac{1}{2 n}, \frac{1}{87}\right)$.

Nevertheless, the conjectures on minorizations of probabilities in (8.1) and (8.3) remain in force.

\section{Problem 9. Conjecture on the determinant of normal matrices}

The so-called determinantal conjecture of Marcus and De Oliveira (OMC in short) can be stated as follows:

$$
\begin{gathered}
\text { Let } A \text { and } B \text { be normal }(n, n) \text { matrices with prescribed } \\
\text { eigenvalues } a_{1}, \ldots, a_{n} \text { and } b_{1}, \ldots, b_{n} \text { respectively; then: } \\
\operatorname{det}(A-B) \in \operatorname{co}\left\{\prod_{i=1}^{n}\left(a_{i}-b_{\sigma(i)}\right): \sigma \text { permutation of }\{1,2, \ldots, n\}\right\} .
\end{gathered}
$$

Normal matrices are complex $(n, n)$ matrices which can be diagonalized via unitary matrices; there are many characterizations of them, more than ninety $[9,19,22,35]$. Hermitian, skew-Hermitian, unitary matrices are examples of normal matrices. Note however that $A-B$ is not necessarily normal even if both $A$ and $B$ are.

The OMC conjecture is known to be true in several situations: when $n \leq 3$; when both $A$ and $B$ are Hermitian; when both $A$ and $B$ are unitary; when $A$ is positive definite and $B$ is skew-Hermitian; when $A$ is positive definite and $B$ is a non-real scalar multiple of a Hermitian matrix; when $A$ is Hermitian and $B$ is a non-real scalar multiple of a Hermitian matrix (see [16] and references therein). For an easy way to grasp the meaning of (9.1), think of the two following specific cases:

- When both $A$ and $B$ are Hermitian (hence with real eigenvalues $a_{1}, \ldots, a_{n}$ and $b_{1}, \ldots, b_{n}$ respectively); then $\operatorname{det}(A-B)$ lies in the line-segment

$$
\left[\min _{\sigma} \prod_{i=1}^{n}\left(a_{i}-b_{\sigma(i)}\right), \max _{\sigma} \prod_{i=1}^{n}\left(a_{i}-b_{\sigma(i)}\right)\right],
$$

where the min and the max are taken over all the permutations $\sigma$ of $\{1,2, \ldots, n\}$ ([9], Thm. VI.7.1). 
- When $A$ and $B$ commute: in that case, there is an unitary matrix which diagonalizes both $A$ and $B$, so that $\operatorname{det}(A-B)$ indeed equals $\prod_{i=1}^{n}\left(a_{i}-b_{\sigma(i)}\right)$ for some permutation $\sigma$ of $\{1,2, \ldots, n\}$.

We began our paper with a statement, let us end it with another one: "One does, by openly facing a well known unsolved problem, run the risk of being remembered more by one's failure than anything else"; attributed to G. Choquet (1915-2006) by A. Connes in his paper in Mathematics: frontiers and perspectives (2000, by the International Mathematical Union, published by the American Mathematical Society).

\section{REFERENCES}

[1] T. Andreescu, O. Mushkarov and L. Stoyanov, Geometric problems on maxima and minima. Birkhäuser (2006).

[2] M. Atiyah and P. Sutcliffe, The geometry of point particles. Proc. R. Soc. London A 458 (2002) $1089-1115$.

[3] M. Atiyah and P. Sutcliffe, Polyhedra in physics, chemistry and geometry. Milan J. Math. 71 (2003) 33-58.

[4] R. Bapat, Mixed discriminants of positive semidefinite matrices. Linear Algebra Appl. 126 (1989) $107-124$.

[5] M. Bayart, Épreuve de mathématiques générales du concours d'agrégation de mathématiques 1980. Revue de Mathématiques Spéciales (1980-1981) 220-230.

[6] A. Ben Tal, A. Nemirovski and C. Roos, Robust solutions of uncertain quadratic and conic-quadratic problems. SIAM J. Optim. 13 (2002) 535-560.

[7] E. Bendito, A. Carmona, A.M. Encinas and J.M. Gesto, Estimation of Fekete points. J. Comput. Phys. 225 (2007) $2354-2376$.

[8] D. Bessis, P. Moussa and M. Villani, Monotonic converging variational approximations to the functional integrals in quantum statistical mechanics. J. Math. Phys. 16 (1975) 2318-2325.

[9] R. Bhatia, Matrix analysis. Springer (1997).

[10] J. Bochnak and J. Siciak, Polynomials and multilinear mappings in topological vector spaces. Studia Math. 39 (1971) 59-76.

[11] G-S. Cheon and I.M. Wanless, An update on Minc's survey of open problems involving permanents. Linear Algebra Appl. 403 (2005) 314-342.

[12] H.T. Croft, K.J. Falconer and R.K. Guy, Unsolved problems in geometry. Springer-verlag (1991).

[13] K. Derinkuyu and M. Pinar, On the S-procedure and some variants. Math. Meth. Oper. Res. 64 (2006) 55-77.

[14] K. Derinkuyu, M. Pinar and A. Camci, An improved probability bound for the approximate S-lemma. Oper. Res. Lett. 35 (2007) 743-746.

[15] M. Drmota, W. Schachermayer and J. Teichmann, A hyper-geometric approach to the BMV-conjecture. Monatshefte Math. 146 (2005) 179-201.

[16] S.W. Drury, Essentially Hermitian matrices revisited. Electronic J. Linear Algebra 15 (2006) $285-296$.

[17] G.P. Egorychev, The solution of Van der Waerden's problem for permanents. Dokl. Akad. Sci. SSSR 258 (1981) $1041-1044$ (in Russian), Adv. Math. 42 (1981) 299-305.

[18] G.P. Egorychev, Proof of the Van der Waerden conjecture. Siberian Math. J. 22 (1982) 854-859.

[19] L. Elsner and K.D. Ikramov, Normal matrices: an update. Linear Algebra Appl. 285 (1998) $291-303$.

[20] D.I. Falikman, A proof of the Van der Waerden conjecture on the permanent of a doubly stochastic matrix. Mat. Zametki 29 (1981) 931-938 (in Russian).

[21] M. Fannes and D. Petz, Perturbation of Wigner matrices and a conjecture. Proc. Amer. Math. Soc. 131 (2003) $1981-1988$.

[22] R. Grone, C.R. Johnson, E.M. Sa and H. Wolkowicz, Normal matrices. Linear Algebra Appl. 87 (1987) $213-225$.

[23] L. Gurvits, The Van der Waerden conjecture for mixed discriminants. Adv. Math. 200 (2006) $435-454$.

[24] L. Gurvits, A proof of hyperbolic Van der Waerden conjecture: the right generalization is the ultimate simplification. Preprint (2006).

[25] D. Hägele, Proof of the cases $p \leqslant 7$ of the Lieb-Seiringer formulation of the Bessis-Moussa-Villani conjecture. J. Stat. Phys. 127 (2007) 1167-1171.

[26] O. Hanner and H. Radstrom, A generalization of a theorem of Fenchel. Proceedings of the American Mathematical Society 2 (1951) 589-593.

[27] F. Hansen, Trace functions as Laplace transforms. J. Math. Phys. 47 (2006) 043504.

[28] D.P. Hardin and E.B. Saff, Discretizing manifolds via minimum energy points. Notices Amer. Math. Soc. 51 (2004) $1186-1194$.

[29] S. He, Z.-Q. Luo, J. Nie and S. Zhang, Semidefinite relaxation bounds for indefinite homogeneous quadratic optimization. Technical report, Department of systems engineering and engineering management, the Chinese University of Hong-Kong (2007).

[30] C. Hillar, Advances on the Bessis-Moussa-Villani trace conjecture. Linear Algebra Appl. 426 (2007) 130-142.

[31] C. Hillar and C.R. Johnson, On the positivity of the coefficients of a certain polynomial defined by two positive definite matrices. J. Statist. Phys. 118 (2005) 781-789.

[32] J.-B. Hiriart-Urruty, Potpourri of conjectures and open questions in Nonlinear analysis and Optimization. SIAM Review 49 (2007) 255-273.

[33] J.-B. Hiriart-Urruty and C. Lemaréchal, Convex Analysis and Minimization Algorithms I, Grundlehren der mathematischen Wissenschaften 305. Springer-Verlag (1993); 2nd edition in 1996. 
[34] R. Holzman and D.J. Kleitman, On the product of sign vectors and unit vectors. Combinatorica 12 (1992) 303-316.

[35] R.A. Horn and C.R. Johnson, Matrix analysis. Cambridge University Press (1985).

[36] H.-X. Huang, P. Pardalos and Z.-J. Shen, A point balance algorithm for the spherical code problem. J. Global Optim. 19 (2001) 329-344.

[37] C.R. Johnson and C.J. Hillar, Eigenvalues of words in two positive definite letters. SIAM J. Matrix Anal. Appl. 23 (2002) 916-928.

[38] C.R. Johnson, S. Leichenauer, P. McNamara and R. Costas, Principal minor sums of $(A+t B)^{m}$. Linear Algebra Appl. 411 (2005) 386-389.

[39] H. Joris, Le chasseur perdu dans la forêt : un problème de géométrie plane. Elem. Math. 35 (1980) 1-14.

[40] D. Knuth, A permanent inequality. Amer. Math. Monthly 88 (1981) 731-740.

[41] A.B.J. Kuijlaars and E.B. Saff, Asymptotics for minimal discrete energy on the sphere. Trans. Amer. Math. Soc. 350 (1998) $523-538$.

[42] J.C. Lagarias, The Van der Waerden conjecture: two soviet solutions. Notices Amer. Math. Soc. 29 (1982) 130-133.

[43] E.H. Lieb and R. Seiringer, Equivalent forms of the Bessis-Moussa-Villani conjecture. J. Statist. Phys. 115 (2004) $185-190$.

[44] M. Marcus and M. Newman, On the minimum of the permanent of a doubly stochastic matrix. Duke Math. J. 26 (1959) $61-72$.

[45] H. Minc, Permanents, Encyclopedia of Mathematics and its Applications 6. Addison-Wesley, Reading, Mass (1978).

[46] A. Mouchet, Bounding the ground-sate energy of a many-body system with the differential method. Nuclear Phys. A 765 (2006) 319-341.

[47] A. Mouchet, Upper and lower bounds for an eigenvalue associated with a positive eigenvector J. Math. Phys. 47 (2006) 022109.

[48] P. Moussa, On the representation of $\operatorname{Tr}\left[\mathrm{e}^{(A-\lambda B)}\right]$ as a Laplace transform. Rev. Math. Phy. 12 (2000) 621-655.

[49] P.J. Nahin, When least is best. Princeton University Press (2004).

[50] Y. Nesterov and A. Nemirovski, Interior-point polynomial algorithms in convex programming. SIAM Studies in Applied Mathematics (1994).

[51] D. Niven, Maxima and minima without calculus. Reprinted by the Mathematical Association of America (2006).

[52] J.D. Pinter, Globally optimized spherical point arrangements: model variants and illustrative results. Ann. Oper. Res. 104 (2001) 213-230.

[53] E.A. Rakhmanov, E.B. Saff and Y. Zhou, Minimal discrete energy on the sphere. Math. Res. Lett. 1 (1994) 647-662.

[54] E.B. Saff and A.B.J. Kuijlaars, Distributing many points on the sphere. Math. Intelligencer 19 (1997) 5-11.

[55] S. Smale, Mathematical problems for the next century. Math. Intelligencer 20 (1998) 7-15.

[56] W.J.H. Stortelder, J.J.B. de Swart and J.D. Pinter, Finding elliptic Fekete points sets: two numerical approaches. J. Comput. Appl. Math. 130 (2001) 205-216.

[57] P.L. Takouda, Problèmes d'approximation linéaires coniques : Approches par projections et via Optimisation sous contraintes de semidéfinie positivité. Ph.D. thesis, Paul Sabatier University, Toulouse, France (2003).

[58] J.H. Van Lint, Notes on Egorychev's proof of the Van der Waerden conjecture. Linear Algebra Appl. 39 (1981) 1-8. 